aus der Glocke außerordentlich schnell das Wasser verliert. Schon nach 10 Minuten betrug der Wassergehalt des Linoxyns $1,8^{\circ} /$ und nach 2 Stunden hatte es das ursprungliche Gewicht erreicht. Somit kann das Linoxyn (bei Zimmertemperatur) tatsächlich etwa $15 \%$ Wasser aufnehmen. Es besteht immer ein Gleichgewicht zwischen Wasserdampf und Linoxyn, weIches sich in dünnen Schichten sehr schnell einstellt. Da das Wasser sich in außerordentlich feiner Verteilung befindet, so muß es verseifend auf das Linoxyn einwirken. Je dünner der Film ist und je feuchter die Luft, desto schneller wird die Verseifung vor sich gehen. An und für sich hat der Trockenvorgang mit der Verseifung und der damit verbundenen Săurezahlerhöhung nichts zu tun. Die von Eibners ${ }^{s}$ gefundenen sehr bohen Säurezahlen eines Leinölfilms können keinesfalls mabgebend für den Trockenvorgang des Leinöls sein. Nur unter bestimmten Bedingungen können die Säurezahlen so hoch werden. So stieg z. B. die Säurezahl eines Linoxyns, welches 2 Jahre lang in einer Glasflasche aufbewahrt wurde, von 33 auf 44. Die Säurezahl eines anderen etwa 3 Monate alten Stückes Linoxyn betrug nur 10. Zum Teil wird die Erhöhung der Säurezahl durch Bildung von Săuren mit niedrigem Molekulargewicht verursacht.

Zusammenfassung:

1. Der Trockenvorgang des Leinöls besteht aus einem chemischen ProzeB (Oxydation) und aus einem kolloiden ProzeB (Gelatinierung). 2. Die Gelatinierung wird durch Temperaturerhöhung beschleunigt 3. Die Oxydation der Leinoblfettsäuren verläuft schneller als diejenige des Leinöls. 4. Die Oxydation des Leinöls wird durch Zusatz von Linol- oder Linolensäure beschleunigt. 5 . Die Ölsäure beschleunigt den Gelatinierungsvorgang des Leinöls. 6. Es wurde, übereinstimmend mit der Angabe von Treumann, gefunden, daß das Iinoxyn bis zu $15 \%$ Wasser aufnehmen kann.

[A. 124.]

\section{Über die Säuren des Urans, Wolframs und Molybdäns.}

Von Gustav F. HütTig, Clausthal.

Vorgetragen auf der Hauptversammlung Hamburg am 9. Juni 1022 in der Fachgruppe fur anorg. Chemie.

(Eingeg. 13.j6, 1922.)

Die Trioxyde des Urans, Wolframg und Molybdäns vermögen durch AnIagerung von Wasser Hydrate zu bilden. Diese Hydrate sind feste Stoffe mit verhältnismäßig geringer Wasserloslichkeit und einem ausgesprochenen Săurecharakter; die von diesen Säuren abgeleiteten Salze bilden die große Gruppe der Uranate, Wolframate und Molybdate.

Die in der Literatur angegebenen Hydrate des Uran-, Wolframund Molybdäntrioxyds sind sehr zahlreich. Als Hydrate des Urantrioxyds sind beschrieben: $\mathrm{UO}_{8} \cdot \mathrm{H}_{2} \mathrm{O}$ und $\mathrm{UO}_{3} \cdot 2 \mathrm{H}_{2} \mathrm{O}$, hingegen nicht die den beständigsten Uranaten zugrunde liegende Pyrouransăure $\mathrm{H}_{2} \mathrm{U}_{2} \mathrm{O}_{7}=2 \mathrm{UO}_{3} \cdot \mathrm{H}_{2} \mathrm{O}$. Als Hydrate des Wolframtrioxyds sind angegeben: 2 $\mathrm{WO}_{3} \mathrm{H}_{2} \mathrm{O}$ (Pyrowolframsäure), $\mathrm{WO}_{3} \mathrm{H}_{2} \mathrm{O}$ (gelbe Wolframsăure), $4 \mathrm{WO}_{3} \cdot 7 \mathrm{H}_{2} \mathrm{O}$ (Metaluteowolframsäure), $\mathrm{WO}_{3} \cdot 2 \mathrm{H}_{2} \mathrm{O}$ (weiße Wolframsäure), $4 \mathrm{WO}_{3} \cdot \mathrm{H}_{2} \mathrm{O} \cdot 9 \mathrm{H}_{2} \mathrm{O}$ (Metawolframsäure), $2 \mathrm{WO}_{3} \cdot 5 \mathrm{H}_{2} \mathrm{O}$ sowie eine große Anzahl von Wolframsäuren mit variablem oder unbestimmtem Wassergehalt, zu denen auch die Parawolframsäuren $12 \mathrm{WO}_{3} \cdot 5 \mathrm{H}_{2} \mathrm{O}$ $+x \mathrm{H}_{2} \mathrm{O}$ und die kolloidalen Modifikationen zu zählen sind. Als Hydrate des Molybdäntrioxyds sind bescbrieben $\mathrm{MoO}_{3} \mathrm{H}_{2} \mathrm{O}$ und $\mathrm{MoO}_{3} \cdot 2 \mathrm{H}_{2} \mathrm{O}$ sowie kolloidales Molybdäntrioxydhydrat. Feste Hydrate des hier wegen der chemischen Ahnlichkeit interessierenden Chromtrioxyds sind nicht bekannt.

Eine systematische Untersuchung daräber, welche von diesen Hydraten wirklich stabile Verbindungen sind, ob die bisherigen Literaturangaben keine Lücken aufweisen, unter welchen Bedingungen die einzelnen Hydrate existenzfäbig sind, und wie die Angaben luber den variablen Wassergehalt mit den stöchiomętrischen Forderangen vereinbar sind, ist bisher nicht erfolgt; ebenso sind - ganz im Gegensatz zu den thermochemisch wohl untersuchten Säuren, des Schwefels, Selens und Tellurs, die im periodischen System die Seitenkolonne zu unseren Elementen bilden - die Bildungswärmen dieser Säuren nicht bekannt.

Ich will nun über diejenigen gemeinschaftlich mit E. v. Schroeder und B. Kurre von mir im chemischen Institut der Bergakademie Clausthal ausgeführten Untersuchungen kurz berichten, die sich mit der Beantwortung dieser Fragen befassen ${ }^{1}$ ). Diese Untersuchungen sind durch eine von W. Biltz ins Werk gesetzte Arbeit über die Beständigkeitsgebiete der Uranoxyde angeregt worden.

Es wurden zunächst möglichst wasserreiche Hydrate als Ausgangspräparate dargestellt. Das analysierte und eingewogene Präparat wurde jedesmal dann noch mit einer gemessenen uberschüssigen

8) Eibner, Zum Trockenvorgang fetter Öle. Farbenzeitung, Jabrg. 26, S. $\delta 81$

1) Das vollständige experimentelle Material wird in der Ztschr. f. anorg. u. allg. Chem. bokanntgegeben. Bisher ist erschienen: „Uber die Hydrate des Urantetroxyds und Urantrioxyds" " Ztschr. f. anorg. u. allg. Chem., 121, 243, [1922]: Úber die Hydrate des Wolframtrioxyds" ${ }^{*}$, Ztschr. f. anorg. u. allg. Chem. 122, 44 [1922].
Menge Wasser versetzt und stand unter zeitweiligen Umschutteln mehrere Tage. Dann wurde dieses in einem kleinen, gewogenen Glasröhrchen oder Quarzrohr aufbewahrte und mit einem luftdichten Glashahn abgeschlossene Prăparat durch Öffnen dieses Hahnes mit einem ungefähr 1 Liter fassenden, evakuierten, dem Inhalt nach genau ausgemessenen Raum in Verbindung gebracht; dann wurde die Temperatur des Präparates lang sam gesteigert, bis der manometrisch gemessene Wasserdampfdruck auf $15 \mathrm{~mm}$ (Quecksilber) stieg. Hierauf wurde durch Feststellung der Gewichtsabnahme oder durch Messung der abgegebenen Wassermenge der in dem Präparat verbliebene Wassergehalt bestimmt und dementsprecbend die stöchiometrische Zusammensetzung berechnet. Dann wurde die Substanz im Vakuum neuerdings erwärmt und wieder die Temperatur festgestellt, bei der der Wasserdampfdruck von $15 \mathrm{~mm}$ erreicht war. Auf diese Weise kann man für jedes an das Trioxyd gebundene Wasser bestimmen, bei welcher Zersetzungstemperatur es unter dem stets gleichen Wasserdampfdruck abgegeben wird. Dadurch, daß jede stöchiometrische chemische Verbindung unter solchen Umständen durch eine bestimmte Zersetzungstemperatur gekennzeichnet ist, kann man an dem Auftreten eines Temperaturfixpunktes das Auftreten einer chemischen Verbindung erkennen. Die Zusammensetzung der chemischen Verbindung ergibt sich unmittelbar aus der Feststellung des Wassergehaltes in der eben beschriebenen Weise. Sehr anschaulich lassen sich die Ergebnisse durch ein Diagramm darstellen, wenn man den jeweiligen Wassergebalt der Verbindung (Anzahl Mole $\mathrm{H}_{2} \mathrm{O}$ auf 1 Mol Trioxyd) etwa auf der Ordinatenachse und die zugehörige Zersetzungstemperatur (bei dem konstanten Wasserdampfdruck von $15 \mathrm{~mm}$ ) auf der Abszissenachse aufträgt.

Die einzelnen Trioxydhydrate sind dann in der Weise gekennzeichnet, wie es die Figur zeigt:

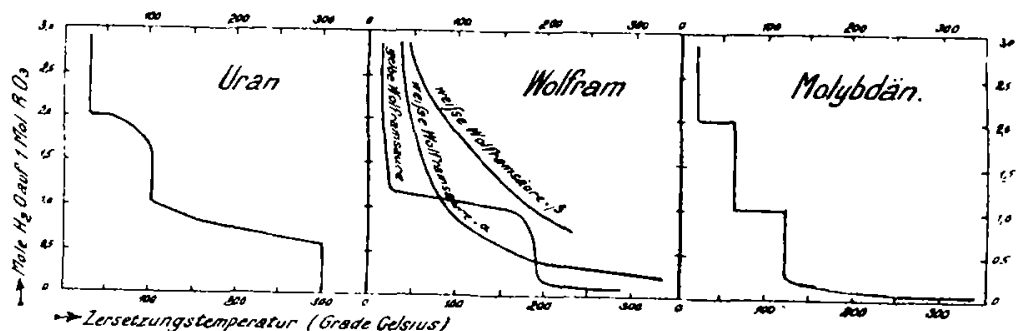

Der Abbau der Urantrioxydhydrate wurde so eingeleitet, daß eine bekannte Menge von sehr reinem Urantrioxyd mit einer bekannten Menge überschüssigen Wassers versetzt wurde. Bei dem Abbau bekam man erst dann von den Werten des reinen Wassers merklich abweichende Drucktemperaturwertpaare, wenn der Wassergehalt entsprechend einer Zusammensetzung $\mathrm{UO}_{3} \cdot 2 \mathrm{H}_{2} \mathrm{O}$ unterschritten wurde. Von da ab wird das erste halbe Mol all mählich abgegeben, weńn man die Temperatur bis $100^{\circ}$ steigert; das $\mathrm{z}$ weite halbe Mol wird dann auf einmal s c harf bei $100^{\circ}$ abgespalten; das dritte halbe Mol wird während des Temperaturanstieges von $100^{\circ}$ auf $300^{\circ}$ wieder allmählich abgegeben, wăhrend das vierte halbe Mol scharf bei $300^{\circ}$ abgegeben wird. Aus dem Diagramm entnimmt man, daß es folgende stabile Hydrate des $\mathrm{UO}_{3}$ gibt: $\mathrm{UO}_{3} \cdot 2 \mathrm{H}_{2} \mathrm{O} \cdot \mathrm{UO}_{3} \cdot 1 \mathrm{H}_{2} \mathrm{O}$ und $\mathrm{UO}_{3} \cdot 0,5 \mathrm{H}_{2} \mathrm{O}$; diese letztere Verbindung hat sich noch ausgeprägter bei einem Abbau der Uranperoxydhydrate ergeben. Eine sichere Entscheidung über Existenz oder Nichtexistenz der Verbindung $\mathrm{UO}_{3} \cdot 1,5 \mathrm{H}_{2} \mathrm{O}$ kann nicht erfolgen.

Bei den Wolframtrioxydhydraten fallen die Ergebnisse verschieden aus, je nachdem, ob man von der gelben oder von der weißen Wolframsäure ausgeht. Die weiße Wolframsăure zeigt während ibres ganzen Abbaues keine Merkmale einer den strchiometrischen Gesetzen unterliegenden chemischen Verbindung; wir müssen sie als typische Adsorptionsverbindung ansprechen. Im Einklang damit steht auch die Tatsache, daß die Festigkeit, mit der sie das Wasser bindet, abbängig ist von dem Dispersitätsgrade der betreffenden Săure. Die als weiße Wolframsäure $=\alpha$ bezeichnete Säure ist weniger feinkörnig als die als weiße Wolframsäure $=: \beta$ bezeichnete Säure; die Filtration der letateren ist selbst durch Membranfilter schwierig; sie hat eine starke Nejgung, sich im Verlauf des Abbaues gelb zu färben. - Im Gegensatz zu der weißen Wolframsäure zeigt die gelbe Wolframsäure deutlich das Auftreten einer chemischen Verbindung $\mathrm{WO}_{3}+1 \mathrm{H}_{2} \mathrm{O}$. Die bei dieser Säure auftretenden Zersetzungswerte sind streng reproduzierbar, was auch daraus hervorgeht, daß die in dieser Kurve sich zwanglos ergänzenden Punkte von zwei verschiedenen unter etwas veränderten Umständen erfolgten Versuchsreihen herrühren. Nur bei einem Wassergehalt unterhalb 0,25 Molen $\mathrm{H}_{2} \mathrm{O}$ und wohl auch oberhalb $1 \mathrm{Mol} \mathrm{H}_{2} \mathrm{O}$ auf $1 \mathrm{Mol} \mathrm{WO}$ zeigt auch die gelbe Wolframsäure den Charakter einer Adsorptionsverbindung. Ich bin der Ansicht, daß hier ein doppeltes therschneiden der Existenzgebiete einer Adsorptionsverbindung mit dem Existenzgebiete einer rein chemischen Verbindung vorliegt. Als einzige feste, stabile, echte chemische Verbindung besteht demnach bei dem Wolframtrioxyd das $\mathrm{WO}_{3} \mathrm{H}_{2} \mathrm{O}$ ( $\mathrm{Zer}$ setzungstemperatur etwa $187^{\circ}$ bei $\mathrm{p}_{2} \mathrm{O}=14 \mathrm{~mm}$ ), hingegen konnte nirgends ein Anhaltspunkt über die stabile Existenz des in der Literatur als nach stöchiometrischen Gesetzen zusammengesetzt angegebenen $\mathrm{WO}_{3} \cdot 2 \mathrm{H}_{2} \mathrm{O}$ gewonnen werden. 
Als Ausgangsprodukt für den Ahbau der Molybdäntrioxydhydrate wurde dasjenige benutzt, das sich nach mehrmonatlichem Stehen einer salpetersauren Ammoniummolybdatiosung aus dieser ausscheidet. Man sieht hiel scharf wie an einem Schulbeispiel das Auftreten der Verbindungen $\mathrm{MoO}_{3} \cdot 2 \mathrm{H}_{2} \mathrm{O}$ (Zersetzungstemperatur $=61^{\circ}$ bei $\mathrm{p} \mathrm{H}_{2} \mathrm{O}=19 \mathrm{~mm}$ ) und $\mathrm{MoO}_{: 3} \mathrm{H}_{2} \mathrm{O}$ (Zersetzungstemperatur = $120^{\circ}$ bei $\mathrm{p} \mathrm{H}_{\mathrm{u}} \mathrm{O}=17 \mathrm{~mm}$ ). Aber auch hier wird der letzte Wassergehalt (unterhalb 0,3 Molen $\mathrm{H}_{3} \mathrm{O}$ auf 1 Mol $\mathrm{MoO}_{3}$ ) nach Art einer Adsorptionsverbindung festgehalten.

Auch die Hydrate des Chromtrioxyds wurden mit unseren experimentellen Methoden untersucht, ohne das 3 aber hierbel - in Übereinstimmung mit den Angaben der Literatur - überbaupt $\mathbf{z u}$ irgendwelchen festen stabilen Hydraten zu gelangen war.

Da es sich bei allen diesen Untersuchungen um ein systematisches Absuchen der Existenzgebiete handelt, ist die Annabme gerechtfertigt, da $\beta$ es andere freie, feste, stabile Hydrate der Trioxyde des Urans, Wolframs, Molybdäns und Chroms als die eben mitgeteilten nicbt gibt. Durch die Feststellung der Existenzgebiete der einzelnen Hydrate sind auch die Affinitäten dieser Verbindungen gegeben; sie sind bei konstantem Druck proportional den Zersetzungstemperaturen, also unmittelbar aus unserem Diagramm ablesbar.

Insoweit sich die Abbaureaktionen unmittelbar den thermodynamischen Gesetzen fügen, ist auf Grund der Nernstschen Näherungsformel auch die Berechnung der Bildungswärmen der einzelnen Hydrate moglich. Es berechnet sich auf dieser Grundlage:

$$
\begin{aligned}
& \mathrm{UO}_{3}+1 / 2 \mathrm{H}_{2} \mathrm{O}=\mathrm{UO}_{3} 1 / 2 \mathrm{H}_{2} \mathrm{O}+13290 \mathrm{cal} \text {, } \\
& \mathrm{UO}_{3}+\mathrm{H}_{2} \mathrm{O}=\mathrm{UO}_{3} \mathrm{H}_{2} \mathrm{O}+23400 \\
& \mathrm{UO}_{3}+11_{2} \mathrm{H}_{2} \mathrm{O}=\mathrm{UO}_{3} 1_{1 / 2} \mathrm{H}_{2} \mathrm{O}+31740 \\
& \mathrm{UO}_{3}+2 \mathrm{H}_{2} \mathrm{O}=\mathrm{UO}_{3} \quad 2 \mathrm{H}_{2} \mathrm{O}+39240 \text { " } \\
& \mathrm{WO}_{3}+\mathrm{H}_{2} \mathrm{O}=\mathrm{WO} \mathrm{H}_{2} \mathrm{O}+20950 \text {. } \\
& \mathrm{MoO}_{3}+\mathrm{H}_{2} \mathrm{O}=\mathrm{MoO}_{3} \cdot \mathrm{H}_{2} \mathrm{O}+18760
\end{aligned}
$$

\section{Über vergleichende Pökelversuche von Fleisch unter Zusatz von Salpeter und Natriumnitrit zur Lake.}

AnläBlich der Bemerkungen von Dr. F. Auerbach und Dr. G. Riel3. Von Dr. Leopold Pollak, Aussig (Elbe).

\section{(Eingeg. 14./6. 1922.)}

Im Anschluß an meine obengenannte Abhandlung (S. 229 dieser Zeitschr, haben Auerbach und $\mathrm{RieB}$ einige Bemerkungen veröffentlicht, die beantwortet werden müssen. Ich stehe nach wie vor auf dem Standpunkt, daß meine Versuchsreihen sich zu jenen von Au erb a ch-Rie $B$ im Widerspruch befinden. Erstens betrifft dies die von mir festgestellte weitgehende Zersetzung des Salpeters, zweitens die immer beobachtele konstante A bnahme des Nitritgehaltes bei der Nitritpökelung. Die in Tabelle II enthaltene ganz geringe Zunahme des Nitritgehaltes zu Beginn des Prozesses ist so gering, daB sie auch irgendwelchen Fehlerquellen zugeschrieben werden kann. In den Versuchsreihen von Auerbach und RieB ist dagegen eine fortwährende bedeutende Steigerung des Nitritgehaltes zu bemerken, mit Ausnahme in Tabelle II, auf deren abweichende Resultate Auerbach-Rieß auch hinweisen. In diesem einzigen Fall wăre eine gewisse Úbereinstimmung zu verzeichnen. Die zum Vergleich herangezogenen Werte, nämlich die Höchstwerte, sind aus dem soeben Gesagten nicht verwendbar, da sie sich auf verschiedene Zeitpunkte des Pokelprozesses bezieben. So ist der Wert 14,6 mg bei A uerbach-Ries vom zweiten Pökeltag mit meinem Wert 6,7 mg vom 13. Tag, die A uerbach-RieB Werte 24,5 mg und 45,1 mg beide vom 22. Tag mit meinem Wert $21,3 \mathrm{mg}$ vom 5 . Tag und $27,6 \mathrm{mg}$ vom 2 . Tag verglichen, demnach nicht übereinstimmend. Mein Wert $6,7 \mathrm{mg}$ aus Tabelle II ist weiter oben allgemein besprochen worden. Es ist selbstverständlich, daß ein so verwickelter Prozeß, wie es der PokelprozeB ist, nicht mit zwei Abhandlungen abgetan ist, je mehr darüber gearbeitet wird, um so sicherer wird das wirklich Richtige gefunden werden. Meine Versuchsreihen wurden wie bereits des ofteren erwähnt, und was Tabellen I-III anbelangt mit groBtmöglicher Anlehnung an die Gebräuche der Praxis einer hierzulande hoch entwickelten Industrie durchgeführt, und sind schon aus diesem Grunde nicht nur theoretisch zu behandeln. Ich glaube, daß Schweinefleisch viel mehr gepökelt wird als Rindfleisch, wenigstens in Europa. Auch die Höhe des Salpeterzusatzes gehört in die hier zu erörternden Fragen; die knappe Bemessung des Salpeters auf Grund des Rundschreibens der Reichsfleischstelle vom 3.9.1917 war selbstredend in der Kriegszwangslage notwendig, und wird heute wohl überholt sein. Wenn nun Abänderungen der Versucbsbedingungen, die beim praktischen Pokeln leicht vorkommen können und tatsächlich auch vorkommen, derartige Unterschiede in den Resultaten ergeben, ist die Frage wohl berechtigt und erörterungsfähig, ob die Grundlagen der genannten Verordnungen und deren Durcbfủhrungsbestimmungen heute noch so beurteilt werden dürfen wie zur Zeit ihres Erscheinens. Wenn aber, nach Auerbach-RieB, die ZweckmäBigkeit der deutschen Verordnungen, die den Gebrauch salpetrigsaurer Salze bei Herstellung von Pokelfleisch verbieten, im Rahmen dieser Zeitschrift nicht erörtert werden soll, so wäre es doch erwünscht, wenn Auerbach-Rie ihren Standpunkt zu dem von mir auf Seite 232 vorletzter Absatz angezogenen Vergleich, gegrünte (gekupferte) Gemüsekonserven betreffend, präzisieren wollten, der mir gut genug erscheint, um bier erörtert zu werden. [A. 157.]

\section{Personal- und Hochschulnachrichten.}

Dr. F. v. Soxleth, Prof. der Agrikulturcbemie an der Technischen Hochschule München, feierte am 20. Juli sein fünfzigjähriges Doktorjubiläum.

Ehrungen: Fabrikant L. Grötzinger, Gründer und langiăhriger Mitinhaber der Süddeutschen Öl- und Melanolwerke G. m. b. H. Freiburg i. Br., wurde auf Grund seiner Verdienste um die Forderung der Wissenschaft zum Dr. phil. h. c. der Universität Freiburg ernannt G. E. Junius, Direktor der Dr. C. Otto \& Co., G. m. b. H., Dahlhausen/Ruhr, ist in Anerkennung seiner Verdienste um die Entwicklung der Nebenproduktenkokerei und des Koksofenbaues von der Technischen Hochschule Karlsruhe die Würde eines Dr.-Ing. E. h. Geh. Rat Dr. phil. Dr. med. h. c. Willstätter, München, ist die Würde eines Dr.-Ing. F. h. von der Technischen Hochschule Darmstadt verliehen worden.

Die Professoren der Physik Dr. H. K. Onnes an der Universität Leiden, Dr. H. Zeemann an der Universität Amsterdam und Dr. $\mathrm{N}$. Bohr an der Universität Kopenhagen wurden zu korrespondierenden Mitgliedern der physikalisch-matbematischen Klasse der Preußischen Akademie der Wissenschaften gewählt.

Es wurden berufen: Dr. H. Hamburger auf die bisher von Prof. Schur innegehabte außerordentliche Professur fur Mathematik an der Universität Berlin; Dr. H. Meerwein, a. o. Prof. in Bonn, auf den durch die Emeritierung des Geh. Reg.-Rats H. Klinger erledigten Lehrstuhl der Chemie an der Universität Konjgsberg; Dr. C. Siegel, Privatdozent an der Universität Göttingen, auf das Ordinariat der Mathematik an der Universität Frankfurt a. M. als Nachfolger von Prof. A. Schönfließ; Dr. H. Schröder, o. Prof. an der Universität Kiel, zum Nachfolger Prof. Tischlers auf den Lehrstuhl der Botanik an der Landwirtschaftlichen Hochschule zu Hohenheim; Dr. O. Tor n a u, als o. Prof. zum Nachfolger von Prof. v. Seelhorst auf den Lehrstuhl für landwirtschaftliche Pflanzenproduktionslehre sowie zur Leitung des landwirtschaftlichen Versuchsfeldes an der Universität Göttingen. Gestorben sind: Chemiker F. Kubrt zu Brakei am 15. Juni im 59. Lebensjahre. - Geh.-R. R. Prof. Dr. H. Rubens, Direktor des Physikalischen Instituts an der Universität Berljn, am 18.6. im 58. Lebensjahre. - Dr. K. Schaefer, Prof. der Chemie u. Abteilungsvorstand am chemischen Laboratorium an der Universität Leipzig, am 17. Juli im 48. Lebensjahre. - Dr. F. Streintz, o. Prof. der Physik an der Technischen Hochschule Graz und Privatdozent an der Universität, im 66. Lebensjahre.

\section{Verein deutscher Chemiker.}

\section{Aus den Bezirksvereinen.}

Bezirksverein Württemberg. Sitzung am 16.6. Vors.: Dr. Sieber. Der Vorsitzende berichtete zunächst über den Verlauf der Hauptversammlung und erteilte sodann das Wort Herrn Prof. Dr. Sauer zu einem Vortrag uber ,;Das Polarisationsmikroskop in seiner Bedeutung für wissenschaftliche und angewandte Chemie".

In der Einleitung wies der Vortr. auf die ja allgemeine Verwendung des gewornhlichen Mikroskopes auch in der Chemie hin, hob aber den grundlegenden Unterschied zwischen diesem und dem Polarisationsmikroskop bervor. Wäbrend das gewöhnliche Mikroskop nur Vorhandenes größier zeigt, deckt das Polarisationsmikroskop die bei gewöhnlicher Vergrößerung unsichtbare Struktur und damit meist auch chemische Unterschiede in kristallisierten Gemischen natürlicher oder ktinstlicher Bildung auf. An einem Beispiel zeigte der Vortr., daß ein Kristall, der unter dem geworhnlichen Mikroskop durchaus einheitlich aussah, sich unter dem Polarisationsmikroskop solort sowohl strukturell als auch chemisch uneinheitlich erwies, ein Grund für nicht aufgeklärte Analysenunterschiede bei scheinbar identischem Ausgangsmaterial. An einigen projizierten Granitdlinnschliffen zeigte Prof. Sauer den mühelosen Nachweis von Zirkon- und Titanverbindungen, sowie die optischen Veränderungen, durch die sich ein Gehalt an radioaktiver Substanz verrät. Durch die weite Verbreitung des Granites, damit des Zirkons und der radioaktiven Substanz ist es erklärlich, daB itberall im Grundwasser Emanation nachweisbar ist, da die meisten Sedimente aus der Zerstörung von Graniten und granitähnlichem Gestein hervorgegangen sind und es demgemä 3 wiederum mikroskopisch-optisch unschwer gelingt, Zirkon als Bestandteil des Sedimentes und Träger der radioaktiven Substanz nachzuweisen. Sehr interessant war es, wie der Vortr. an Hand der gezeigten Dünnschliffe mittels des Polarisationsmikriskops die Entscheidung und die weiteren Schicksale der verschiedenen Gesteinsarten erläuterte. Eine Reihe prächtiger und anschaulicher Dtinnschliffprojektionen im gew öhnlichen und polarisierten Licht beschlob den Vortrag.

Die zablreich erschienenen Zuhorer waren mit der Forderung des Redners einig, dab zur Ausbildung des Chemikers auch das Vertrautsein mit dem Polarisationsmikroskop gehöre. 